\title{
Perfeccionismo socialmente prescrito y los cinco grandes rasgos de la personalidad en niños españoles
}

\author{
María Vicent ${ }^{1}$, Cándido J. Inglés ${ }^{2}$, Carolina Gonzálvez ${ }^{1}$, Ricardo Sanmartín ${ }^{1}$ y \\ José Manuel García-Fernández ${ }^{1}$ \\ ${ }^{1}$ Universidad de Alicante (España); ${ }^{2}$ Universidad Miguel Hernández (España)
}

\begin{abstract}
El objetivo de este estudio consistió en examinar la relación entre el Perfeccionismo Socialmente Prescrito (PSP) y los cinco grandes rasgos de la personalidad en una muestra compuesta por 804 alumnos de Educación Primaria entre 8 y 11 años de edad $(M=9.57$; $S D=1.12)$. Se empleó la subescala PSP de la Child and Adolescent Perfectionism Scale (CAPS) y el Cuestionario Big Five de Personalidad para Niños (BFQ-N) que mide los rasgos de Neuroticismo, Extraversión, Apertura a la experiencia, Cordialidad y Conciencia. Los resultados del análisis de diferencia de medias mostraron que los alumnos con alto PSP puntuaron significativamente más alto en Conciencia, Cordialidad, Extraversión y Apertura a la experiencia que sus iguales con bajos niveles de PSP, con tamaños del efecto pequeños en todos los casos. Por el contrario, no se observaron diferencias significativas en Neuroticismo. Los análisis de regresión logística revelaron que todos los rasgos de la personalidad, a excepción del Neuroticismo, cuyos resultados no alcanzaron la significación estadística, predijeron significativa y positivamente altas puntuaciones en PSP, con niveles de $O R$ que oscilaron entre 1.01 (para Conciencia y Cordialidad) y 1.03 (para Apertura a la experiencia y Extraversión).
\end{abstract}

Palabras clave: Perfeccionismo socialmente prescrito, personalidad, Big Five, Educación Primaria, infancia.

Socially prescribed perfectioism and personality. The aim of this study was to examine the relationship between Socially Prescribed Perfectionism (SPP) and the Big Five personality traits in a sample of 804 Primary School students between 8 and 11 years old $(M=9.57$; $S D=1.12$ ). The SPP subscale of the Child and Adolescent Perfectionism Scale (CAPS) and the Big Five Questionnaire for Children (BFQ-N), which evaluate the traits of Neuroticism, Extraversion, Openness, Agreeableness, and Conscientiousness, were used. The mean difference analysis showed that students with high levels of SPP scored significantly higher on Conscientiousness, Agreeableness, Extraversion and Openness, with small effect sizes for all cases. In contrast, no significant differences were observed in Neuroticism. Logistic regression analysis revealed that all personality traits, except neuroticism, whose results didn't reach the statistical significance, significantly and positively predicted higher scores on PSP, with OR levels ranging from 1.01 (for Conscientiousness and Agreeableness) to 1.03 (for Openness and Extraversion).

Keywords: Socially prescribed perfectionism, personality, Big Five, Primary Education, childhood.

Correspondencia: Cándido J. Inglés. Departamento de Psicología de la Salud; Universidad Miguel Hernández; Avda de la Universidad, s/n. Edificio Altamira. C.P.: 03202. Elche, Alicante(España); E-mail: cjingles@umh.es 
La producción científica sobre perfeccionismo infantojuvenil no puede compararse en términos de productividad y logro, ni cuantitativa ni cualitativamente, con los estudios llevados a cabo en adultos (Morris y Lomax, 2014). Centrándose mayoritariamente en el ámbito de lo psicopatológico, las investigaciones sobre perfeccionismo infantil y personalidad son escasas. Con el presente trabajo, se pretende profundizar en la relación que existe entre el perfeccionismo socialmente prescrito y los cinco grandes rasgos de la personalidad, con el fin de conocer la forma en que estos constructos se relacionan durante la infancia.

\section{personalidad}

Perfeccionismo Socialmente Prescrito y los cinco grandes rasgos de la

El Perfeccionismo Socialmente Prescrito (PSP) es una de las dos dimensiones, junto con el Perfeccionismo Auto-Orientado, que Flett, Hewitt, Boucher, Davidson, y Munro (2000) atribuyen al perfeccionismo infantil. Se trata de una faceta interpersonal, según la cual el perfeccionismo vendría demandado por las personas significantes del entorno del sujeto, a través de la imposición de expectativas de rendimiento muy altas y la tendencia a juzgar duramente los resultados.

La relación entre el PSP y los cinco grandes rasgos de la personalidad (Extraversión, Cordialidad, Conciencia, Apertura a la experiencia y Neuroticismo) ha sido objeto de análisis por diversos estudios previos (Cuttler y Graf, 2007; De Cuyper, Claes, Hermans, Pieters, y Smits, 2015; Dunkley y Kyparissis, 2008; Hill, McIntire, y Bacharach, 1997; Nathanson, Paulhus, y Williams, 2006; Sherry, Hewitt, Flett, Lee-Baggley, y Hall, 2007; Stoeber, Otto, y Dalbert, 2009).

Hill et al. (1997) fueron los primeros en investigar sobre PSP y el modelo de los cinco rasgos, recurriendo a 214 universitarios estadounidenses $(M=19 ; S D=1.6)$. Los análisis correlacionales revelaron asociaciones positivas y significativas entre el PSP y el Neuroticismo, así como negativas con Extraversión y Cordialidad. Por el contrario, las correlaciones entre el PSP y los rasgos de Apertura a la experiencia y Conciencia no alcanzaron la significación estadística. Los análisis predictivos mostraron que solamente el Neuroticismo predijo positivamente el PSP, suponiendo el $12 \%$ de la varianza explicada. Posteriormente, Nathanson et al. (2006) obtuvieron que el PSP correlacionó de forma negativa y significativa únicamente con Cordialidad, Conciencia y Neuroticismo, en una muestra de 770 universitarios canadienses.

Seguidamente, Sherry et al. (2007) obtuvieron, igualmente con población universitaria canadiense $(N=350 ; M=19.13 ; S D=1.86)$ que el PSP se asoció de forma significativa y en sentido positivo con el Neuroticismo, así como en sentido negativo con la Extraversión, Cordialidad y Conciencia. Simultáneamente, Cuttler y Graf (2007) demostraron con su estudio, realizado con 141 adultos canadienses entre 18 y 81 años, que el PSP se asocia de forma positiva y significativa con el Neuroticismo, la Apertura a 
la experiencia y la Cordialidad. Por su parte, Dunkley y Kyparissis (2008) tan sólo reportaron correlaciones significativas entre el PSP y el Neuroticismo y la Cordialidad, en sentido positivo y negativo, respectivamente, con 223 participantes canadienses adultos. Asimismo, en población alemana entre 14 y 19 años $(N=214)$ y con datos longitudinales para un intervalo de entre 5 y 8 meses, Stoeber et al. (2009) observaron correlaciones significativamente positivas entre el PSP y el Neuroticismo, así como negativas con Extraversión, Apertura a la experiencia y Cordialidad. No obstante, los análisis de regresión jerárquica revelaron que ninguno de los cinco rasgos de la personalidad predijo cambios longitudinales significativos en el PSP.

Recientemente, De Cuyper et al. (2015) hallaron que el PSP predijo de forma significativa y negativa el Neuroticismo y la Extraversión en una muestra de 959 universitarios belgas $(M=18.45$ años; $S D=1.75)$. Sin embargo, dicha capacidad predictiva no alcanzó la significación con respecto a la Cordialidad, Conciencia y Apertura a la experiencia.

Asimismo, es conveniente mencionar que otros autores han aportado datos sobre la relación entre el PSP y algunos de los cinco grandes rasgos sin evaluarlos todos conjuntamente, especialmente con el Neuroticismo, como es el caso de Brannan y Petrie (2008), Enns, Cox, y Clara, (2005), Miquelon, Vallevrand, Grouzed, y Cardinal (2005) y Sherry y Hall (2009), cuyos resultados del análisis de correlaciones reveló una relación positiva y significativa entre ambos constructos, habiéndose realizado todos estos trabajos con población norteamericana adulta y/o universitaria.

Igualmente, Molnar et al. (2012) y Molnar et al. (2012), respectivamente, con 489 mujeres canadienses diagnosticadas con fibromialgia $(M=48.78 ; S D=10.41)$ y 550 universitarios también canadienses $(M=22.38 ; S D=.81)$, analizaron únicamente las correlaciones entre el PSP y los rasgos de conciencia, Extraversión y Neuroticismo, encontrando asociaciones significativas y negativas para Conciencia y Extraversión, así como en sentido positivo para el Neuroticismo. Contrariamente, O'Connor y O'Connor (2004) reportaron que el PSP no correlacionó de forma significativa con la Conciencia, en 155 universitarias de Reino Unido (entre 19 y 48 años). En la misma línea, Sherry et al. (2010) observaron, en 1258 profesores de psicología norteamericanos $(M=48.06$; $S D=11.27$ ), correlaciones no significativas entre el PSP y la Conciencia pero sí para el caso del Neuroticismo, en sentido positivo. Adicionalmente, los autores contrastaron la validez discriminante del PSP con respecto al Neuroticismo, cuyos resultados apoyaron la idea de que se trata de constructos distintos.

\section{El presente estudio}

La revisión de la literatura pone de manifiesto que, en general, los estudios previos coinciden en identificar que el PSP se relaciona de forma positiva con el Neuroticismo (Brannan y Petrie, 2008; Cuttler y Graf, 2007; Dunkley y Kyparissis, 
2008; Enns et al., 2005; Hill et al., 1997; Miquelon et al., 2005; Molnar et al., 2012; Molnar et al., 2012; Sherry y Hall, 2009; Sherry et al., 2007; Sherry et al., 2010; Stoeber et al., 2009), negativa con la Extraversión (De Cuyper et al., 20015; Hill et al., 1997; Molnar et al., 2012; Molnar et al., 2012; Sherry et al., 2007; Stoeber et al., 2009), y la Cordialidad (Dunkley y Kyparissis, 2008; Hill et al., 1997; Nathanson et al., 2006; Sherry et al., 2007; Stoeber et al., 2009), así como de forma no significativa con la Apertura a la experiencia (De Cuyper et al., 2015; Dunkley y Kyparissis, 2008; Hill et al., 1997; Nathanson et al., 2006; Sherry et al., 2007) y la Conciencia (Cuttler y Graf, 2007; De Cuyper et al., 2015; Dunkley y Kyparissis, 2008; Hill et al., 1997; Sherry et al., 2010; Stoeber et al., 2009). No obstante, estos resultados no son generalizables a población infantil, ya que todos los estudios citados, a excepción del trabajo de Stoeber et al. (2009) que emplea población adolescente, han recurrido a población adulta o estudiantes universitarios. Igualmente, exceptuando tres estudios (De Cuyper et al., 2015; O’Connor y O’Connor, 2004; Stoeber et al., 2009) que utilizan población belga, inglesa y alemana, el resto han recurrido a participantes norteamericanos. Asimismo, respecto a la metodología y diseño de los estudios, la mayoría de los datos ofrecidos provienen de análisis correlacionales, a excepción de De Cuyper et al. (2015), Hill et al. (1997), Sherry et al. (2010) y Stoeber et al. (2009) que incluyen análisis de regresión y/o modelos estructurales.

En consecuencia, el presente estudio tiene como finalidad examinar la relación entre el PSP y los cinco grandes rasgos de la personalidad en alumnos de Educación Primaria entre 8 y 11 años de edad, con el fin de comprobar si los resultados reportados por la literatura previa son generalizables a población infantil y española. Esta finalidad se concreta en los siguientes objetivos: (a) analizar si los alumnos con altos niveles de PSP difieren de sus iguales con bajos niveles de PSP en las puntuaciones medias en: Neuroticismo, Extraversión, Apertura a la experiencia, Cordialidad y Conciencia; y (b), examinar la capacidad predictiva de los cinco grandes rasgos de la personalidad sobre las altas puntuaciones en PSP.

Según la evidencia empírica previa, se espera que:

Hipótesis 1: los participantes con alto PSP puntúen significativamente más alto en Neuroticismo que sus iguales con bajo PSP, así como que el Neuroticismo sea un predictor significativo y positivo de altos niveles en PSP.

Hipótesis 2: los participantes con alto PSP puntúen significativamente más bajo en Extraversión y Cordialidad que sus iguales con bajo PSP, así como que la Extraversión y la Cordialidad sean predictores significativos y negativos de altos niveles en PSP.

Hipótesis 3: los participantes con alto y bajo PSP no difieran significativamente en las puntuaciones medias en Apertura a la experiencia y 
Conciencia, así como que los rasgos de Conciencia y Apertura a la experiencia no sean predictores significativos de altos niveles en PSP.

\section{MÉTODO}

\section{Participantes}

La muestra se seleccionó mediante un muestreo aleatorio por conglomerados en las zonas norte, sur, este y oeste de la provincia de Alicante, quedando seleccionados, de esta forma, un total de 10 colegios públicos de Educación Primaria. En el caso de que en los centros hubiese más de un grupo por curso académico, se escogió uno de estos al azar (desde $3^{\circ}$ hasta $6^{\circ}$ curso). Siguiendo este procedimiento, se consiguió reclutar una muestra compuesta por 982 alumnos, de los cuales únicamente participaron definitivamente en el estudio 804 (rango de edad=8-11 años, $M=9.57 ; S D=1.12$ ), ya que un $4.96 \%$ fueron excluidos por no presentar el consentimiento de sus padres o tutores legales por escrito; un $4.89 \%$, por no tener el nivel de lectura mínimo requerido para cumplimentar las pruebas; un 3.90\%, por ser alumnado repetidor; así como un $4.38 \%$, por errores u omisiones en las respuestas de los test. El $51.7 \%$ de los participantes eran hombres y el $48.3 \%$ mujeres. Respecto a la distribución de la muestra en función de la edad, se obtuvo que un $21.9 \%, 27.1 \%, 22.6 \%$ y $28.4 \%$ de los participantes tenían $8,9,10$ y 11 años, respectivamente. La prueba $\chi^{2}$ de la homogeneidad de la distribución de frecuencias reveló la ausencia de diferencias estadísticamente significativas para los ocho grupos de sexo por edad $\left(\chi^{2}=5.08 ; p=.17\right)$.

\section{Instrumentos}

The Child and Adolescent Perfectionism Scale (CAPS; Castro et al., 2004; Flett et al., 2000).

Se utilizó la subescala Perfeccionismo Socialmente Prescrito (PSP) del CAPS. Se trata de una dimensión compuesta por 10 ítems valorados a través de una escala tipo Likert de 5 puntos cuyo objetivo es medir la tendencia de los niños y adolescentes (a partir de 8 años) a creer que los demás tienen unas elevadas expectativas y son demasiado críticos con el rendimiento de uno mismo (e.g., "El resto de la gente piensa que he fracasado si no doy lo mejor de mí en todo momento"). En su validación inicial (Flett et al., 2000), el PSP reportó adecuados índices de consistencia interna y fiabilidad test-retest $\left(\alpha=.81 ; r_{x x}=.74\right)$. Igualmente, respecto a la versión española, Castro et al. (2004) informaron sobre índices de consistencia interna, Alfa de Cronbach, de .87.

En el presente estudio, los niveles de Alfa de Cronbach calculados para el PSP fueron .88 .

Cuestionario Big Five de Personalidad para Niños (BFQ-N; Barbaranelli, Caprara, Rabasca, y Pastorelli, 2003; Carrasco, Holgado, y Del Barrio, 2005). 
El instrumento fue desarrollado originalmente por Barbaranelli et al. (2003), en población italiana entre 9 y 14 años de edad, con el objetivo de evaluar los cinco grandes rasgos de la personalidad durante la infancia tardía. Consta de 65 ítems y una escala tipo Likert de cinco puntos, aplicable a partir de los 8 años de edad. La estructura factorial de la escala consta de los siguientes cinco factores:

- Extraversión: mide la creatividad, el entusiasmo, la asertividad y la confianza en uno mismo (e.g., "Me gusta hablar con los otros")

- Cordialidad: refleja la preocupación y sensibilidad hacia los demás y hacia sus necesidades (e.g., "Me comporto con los demás con mucha amabilidad").

- Conciencia: evalúa la autonomía, el orden, la precisión y el cumplimiento de las normas y los compromisos (e.g., "Respeto las reglas y el orden").

- Neuroticismo: referida a los sentimientos de ansiedad, depresión, descontento e ira (e.g., "Me enfado con facilidad").

- Apertura a la experiencia: valora aspectos intelectuales, principalmente de tipo escolar (e.g., "Cuando el maestro explica algo, lo entiendo enseguida").

En su validación con población española, Carrasco et al. (2005) reportaron adecuados niveles de fiabilidad y estabilidad temporal para un intervalo de una semana ( $\alpha=$ entre .88 y .78, respectivamente, para Conciencia y Neuroticismo; $t_{x x}=$ entre $.84 \mathrm{y}$ .62 , respectivamente, para Conciencia y Cordialidad).

En el presente estudio, se obtuvieron niveles de consistencia interna, Alfa de Cronbach, adecuados para todas las subescalas (.90 para Conciencia, .86 para Apertura, .80 para Extraversión, .94 para Cordialidad y .85 para Neuroticismo).

\section{Procedimiento}

Se realizó una reunión con los responsables de cada centro con el fin de explicarles los objetivos de la investigación y solicitar su participación. Seguidamente, se entregó a los padres o tutores legales de la muestra seleccionada una carta informativa que debían devolver firmada en el caso de que dieran su consentimiento para participar en el estudio. Las pruebas se administraron en horario escolar (durante aproximadamente 45 minutos), por grupos y bajo la supervisión de un investigador, el cual insistió en el carácter voluntario de su participación. Se asignó una clave numérica a cada alumno con el fin de garantizar su anonimato.

\section{Análisis de datos}

Se utilizó la prueba $t$ de Student para examinar las diferencias en las dimensiones del BFQ-N entre el grupo con alto PSP (puntuaciones $\geq$ al centil 75) y bajo PSP (puntuaciones $\leq$ al centil 25). Asimismo, se empleó el índice $d$ (diferencia de media tipificada) para comprobar el tamaño del efecto de dichas diferencias: pequeño (valores 
entre .20 y .50), moderado (valores entre .51 y .79) y grande (valores $\geq .80$ ) (Cohen, 1988).

En segundo lugar, se recurrió al método de regresión logística binaria siguiendo el procedimiento de regresión por pasos hacia delante, basado en el estadístico de Wald, para examinar la capacidad predictiva de las dimensiones del BFQ-N, sobre las altas puntuaciones en PSP (puntuaciones $\geq$ al centil 75). Para interpretar los resultados, se atendió al estadístico $O R$, según el cual, puntuaciones $>1$ indican predicción en sentido positivo; <1, en sentido negativo; y =1, que no hay predicción (De Maris, 2003).

Se utilizó el SPSS 20 para realizar todos los análisis estadísticos.

\section{RESULTADOS}

La tabla 1 muestra los resultados para las diferencias de medias entre los participantes con alto y bajo PSP. Tal y como se observa, el grupo con alto PSP puntuó significativamente más alto que el grupo con bajo PSP en todas las dimensiones de la personalidad, exceptuando Neuroticismo, cuyas diferencias no alcanzaron la significación estadística. Respecto a la magnitud de dichas diferencias, se encontraron tamaños del efecto pequeños en todos los casos, que oscilaron entre $d=.20$ y .35 .

Tabla 1. Medias, desviaciones típicas y tamaño del efecto para los cinco grandes rasgos de la personalidad obtenidas por los grupos alto PSP y bajo PSP

\begin{tabular}{lccccccccc}
\hline & \multicolumn{2}{c}{$\begin{array}{c}\text { Prueba } \\
\text { Levene }\end{array}$} & \multicolumn{2}{c}{ Grupo Alto PSP } & \multicolumn{2}{c}{$\begin{array}{c}\text { Grupo Bajo PSP } \\
\text { 346 }\end{array}$} & \multicolumn{3}{c}{$\begin{array}{c}\text { Sig. Estadística y } \\
\text { magnitud de diferencias }\end{array}$} \\
\hline $\begin{array}{l}\text { Dimensiones } \\
\text { del BFQ-N }\end{array}$ & $F$ & $p$ & $M$ & $D E$ & $M$ & $D E$ & $t 802$ & $p$ & $d$ \\
\hline Extraversión & 4.02 & .08 & 46.50 & 10.12 & 43.19 & 8.80 & -4.85 & $<.001$ & .35 \\
\hline Cordialidad & 4.83 & .09 & 49.10 & 11.77 & 46.23 & 12.81 & -3.30 & .001 & .23 \\
\hline Conciencia & .012 & .91 & 47.91 & 11.05 & 45.64 & 11.22 & -2.85 & .004 & .20 \\
\hline Neuroticismo & 3.78 & .06 & 34.94 & 10.28 & 33.58 & 11.45 & -1.75 & .079 & - \\
\hline $\begin{array}{l}\text { Apertura a la } \\
\text { experiencia }\end{array}$ & 3.97 & .07 & 47.37 & 10.05 & 44.08 & 9.52 & -4.70 & $<.001$ & .33 \\
\hline
\end{tabular}

Los resultados de las regresiones logísticas binarias pueden observarse en la tabla 2. El porcentaje de casos correctamente clasificados rondó entre el $69.2 \%$ (para Extraversión) y el $67 \%$ (para Apertura a la experiencia). Igualmente, la $R^{2}$ de Nahelkerke osciló entre .10 (para Apertura a la experiencia) y .06 (para Cordialidad). Los resultados revelaron que todos los rasgos de la personalidad, a excepción del Neuroticismo, cuyos resultados no fueron significativos, predijeron significativa y positivamente altas puntuaciones en PSP. Concretamente, los niveles de $O R$ indicaron que por cada punto que se incrementan las puntuaciones en Extraversión, Cordialidad, Conciencia y Apertura a la experiencia, aumenta, respectivamente, un $3 \%$, un $1 \%$, un $1 \%$ y un $3 \%$, la probabilidad de presentar altos niveles en PSP. 
VICENT et al. Perfeccionismo socialmente prescrito y personalidad

Tabla 2. Regresión logística binaria para la probabilidad de presentar alto PSP en función de las dimensiones del BFQ-N

\begin{tabular}{|c|c|c|c|c|c|c|c|c|c|}
\hline Variable & & $\chi^{2}$ & $\mathrm{R}^{2}$ & B & E.T. & Wald & $p$ & $O R$ & I.C. $95 \%$ \\
\hline \multirow[t]{2}{*}{ Extraversión } & $\begin{array}{c}\text { Clasificados } \\
\text { correc.: } 69.2 \%\end{array}$ & 23.14 & .08 & .03 & .01 & 22.34 & $<.001$ & 1.03 & $1.02-1.05$ \\
\hline & Constante & & -1.32 & & .34 & 14.65 & $<.001$ & .26 & \\
\hline \multirow[t]{2}{*}{ Cordialidad } & $\begin{array}{l}\text { Clasificados } \\
\text { correc.: } 68 \%\end{array}$ & 10.75 & .06 & .01 & .01 & 10.64 & .001 & 1.01 & $1.00-1.03$ \\
\hline & Constante & & -.62 & & .28 & 4.78 & .029 & .53 & \\
\hline \multirow[t]{2}{*}{ Conciencia } & $\begin{array}{c}\text { Clasificados } \\
\text { correc.: } 68.7 \%\end{array}$ & 20.89 & .09 & .01 & .01 & 8.03 & .005 & 1.01 & $1.00-1.03$ \\
\hline & Constante & & -.56 & & .31 & 3.42 & .064 & .56 & . \\
\hline \multirow[t]{2}{*}{$\begin{array}{l}\text { Apertura a la } \\
\text { experiencia }\end{array}$} & $\begin{array}{l}\text { Clasificados } \\
\text { correc.: } 67 \%\end{array}$ & 21.70 & .10 & .03 & .01 & 21.03 & $<.001$ & 1.03 & $1.02-1.04$ \\
\hline & Constante & & -1.26 & & .34 & 13.55 & $<.001$ & .28 & \\
\hline
\end{tabular}

\section{DISCUSIÓN Y CONCLUSIONES}

Este trabajo se propuso examinar la relación entre el PSP y los cinco grandes rasgos de la personalidad en población infantil. Los resultados demostraron que los participantes que reportaron altos niveles de PSP puntuaron significativamente más alto en Extraversión, Cordialidad, Conciencia y Apertura a la experiencia que sus compañeros con bajos niveles de PSP. Asimismo, se obtuvo que tanto Extraversión, Cordialidad, Conciencia y Apertura a la experiencia predijeron significativamente y en sentido positivo altos niveles de PSP. Estos resultados indican que existe una relación positiva entre el PSP y los cuatro rasgos de la personalidad mencionados (i.e., Extraversión, Cordialidad, Conciencia y Apertura a la experiencia), contradiciendo la literatura previa al respecto, la cual ha hallado mayoritariamente apoyos sobre una relación negativa entre el PSP y la Extraversión (De Cuyper et al., 20015; Hill et al., 1997; Molnar et al., 2012; Molnar et al., 2012; Sherry et al., 2007; Stoeber et al., 2009) y la Cordialidad (Dunkley y Kyparissis, 2008; Hill et al., 1997; Nathanson et al., 2006; Sherry et al., 2007; Stoeber et al., 2009), así como resultados no significativos, para los rasgos de Conciencia (Cuttler y Graf, 2007; De Cuyper et al., 2015; Dunkley y Kyparissis, 2008; Hill et al., 1997; Sherry et al., 2010; Stoeber et al., 2009) y Apertura a la experiencia (De Cuyper et al., 2015; Dunkley y Kyparissis, 2008; Hill et al., 1997; Nathanson et al., 2006; Sherry et al., 2007). Ciertamente, cabe mencionar que algunos trabajos previos constituyen excepciones a estos resultados predominantes en la literatura. Así, por ejemplo, Cuttler y Graff (2007) encontraron asociaciones significativas y positivas entre el PSP y la Apertura a la experiencia y la Cordialidad, en consonancia con nuestro trabajo.

Contrariamente a lo esperado, se obtuvo que los participantes con alto y bajo PSP no difirieron significativamente en los niveles de Neuroticismo. Estos resultados fueron sustentados por los análisis de regresión, según los cuales el Neuroticismo no resultó ser un predictor significativo de las altas puntuaciones en PSP, lo cual contradice los hallazgos de una gran cantidad de estudios previos que defienden que ambas variables se asocian significativa y positivamente (Brannan y Perie, 2008; Cuttler y Graf, 2007; Dunkley y Kyparissis, 2008; Enns et al., 2005; Hill et al., 1997; Miquelon et al., 2005; Molnar, Flett et 
al., 2012; Molnar, Sadava et al., 2012; Sherry y Hall, 2009; Sherry et al., 2007; Sherry et al., 2010; Stoeber et al., 2009). Aunque también cabe mencionar que algunos trabajos reportaron asociaciones negativas (De Cuyper et al., 2015; Nathanson et al., 2006). Similarmente, y pese a que el análisis correlacional previo verificó la asociación positiva entre PSP y Neuroticismo, Stoeber et al. (2009) no encontraron evidencias que apoyaran la idea de que el Neuroticismo juega un rol importante en el desarrollo de esta dimensión perfeccionista.

Estas divergencias entre los resultados del presente estudio y la literatura en general pueden deberse a las características de la población empleada, ya que este es el primer trabajo, para nuestro conocimiento, que analiza el PSP y los cinco grandes rasgos de la personalidad en niños y en población española. En este sentido, se ha demostrado que existen divergencias en la forma en que los aspectos culturales influyen en el PSP (Perera y Chang, 2015). Asimismo, es posible que, durante la infancia, el PSP no sea una faceta tan desadaptativa como ha demostrado ser en edades posteriores (e.g., Cox y Chen, 2014; Inglés, Gonzálvez, García-Fernández, Vicent, y Martínez-Monteagudo, 2015; Sherry, Sherry, Hewitt, Mushquash, y Flett, 2015; Stoeber, 2014).

Respecto a la relación entre el PSP y la Cordialidad y la Extraversión, dado el carácter interpersonal de dicha dimensión perfeccionista, no es de extrañar que se asocie con mayores niveles de preocupación por los demás y con un elevado interés por conocer sus sentimientos y creencias, especialmente aquellos que atañen a uno mismo.

Además, la relación entre la Apertura a la experiencia y el PSP podría justificarse por el hecho de que, durante la infancia, la percepción de un entorno exigente con elevadas expectativas no suponga una amenaza para el bienestar del sujeto, sino que más bien le dote de autoconfianza y motivación, repercutiendo positivamente en determinados aspectos de la vida, como, por ejemplo, el rendimiento académico. De hecho, las expectativas paternas se consideran un importante predictor del logro escolar (Álvarez et al., 2015; Castro et al., 2015; Regueiro et al., 2015; Valle et al., 2015). Esto explicaría por qué los alumnos con elevados niveles de PSP puntúan más alto en Apertura a la Experiencia, pues se trata de una dimensión que evalúa, fundamentalmente, aspectos intelectuales relacionados con el rendimiento académico. Igualmente, puede que, durante la infancia, considerar a los padres o maestros como exigentes y duros propicie que los niños sean más meticulosos, organizados y comprometidos con las normas impuestas, con el fin de satisfacer las demandas del entorno y evitar las críticas y castigos. Esta idea estaría en consonancia con la hipótesis de reactividad social (Flett, Hewitt, Oliver, y Mcdonald, 2002), que defiende que el perfeccionismo se desarrolla, en ocasiones, en un intento por escapar de un entorno hostil o unas prácticas de crianza autoritarias.

Finalmente, es posible que la relación entre el PSP y el Neuroticismo sea más significativa en los cursos posteriores en los que el aumento de materias escolares y la dificultad de las mismas suponga que los alumnos empiecen a tener problemas para satisfacer las demandas del entorno y superar el elevado listón que se les ha impuesto, con las consecuentes críticas y reprimendas que ello conlleva, provocando en el sujeto sentimientos de angustia y frustración típicos del Neuroticismo. 
No obstante, conviene recalcar que este trabajo tiene diversas limitaciones. En primer lugar, no es posible establecer relaciones causa-efecto entre los constructos analizados, aspecto que debería abordarse en el futuro empleando datos longitudinales y/o recurriendo a la metodología de ecuaciones estructurales. En segundo lugar, este estudio se ha basado en la conceptualización del perfeccionismo infantil defendida por Flett et al. (2000), y, por tanto, los resultados deben interpretarse en base a dicha perspectiva. No obstante, considerando la gran variedad de medidas y conceptualizaciones que existen sobre el perfeccionismo (véase Stairs, Smith, Zapolski, Combs, y Settles, 2012, para una revisión), convendría comprobar si se replican estos resultados empleando otras subescalas que evalúen constructos similares (e.g, Expectativas Paternas de la Childhood Multidimensional Perfectionism Scale; Dekryger, 2005). Asimismo, las conclusiones del presente estudio no son generalizables a otras muestras que no se correspondan con alumnado español entre 8 y 11 años. En esta línea, sería conveniente que se llevasen a cabo futuras investigaciones que examinasen la relación entre el PSP y el modelo de los cinco rasgos de la personalidad en población española, así como con niños y adolescentes, con el fin de poder contrastar las conclusiones extraídas de este estudio y dotarlas de mayor validez.

Pese a las limitaciones, los hallazgos de esta investigación ofrecen importantes implicaciones prácticas para la comprensión del PSP durante la infancia, tratándose del primer trabajo que ha analizado la relación entre el perfeccionismo y los cinco grandes rasgos de la personalidad en edades tempranas. Los resultados sugieren que, durante la infancia, el PSP presenta un patrón de asociación con la personalidad adaptativo, contrariando la evidencia empírica previa, según la cual el PSP se relaciona de forma desadaptativa con los rasgos de la personalidad en población adulta o universitaria. Es necesario, por tanto, dedicar mayores esfuerzos a investigar y esclarecer cuál es el curso de desarrollo del perfeccionismo socialmente prescrito. Concretamente, indagar sobre los motivos o las variables que puedan influir en su evolución hacia patrones desadaptativos de asociación con el modelo de los cinco grandes rasgos en etapas posteriores a la niñez.

\section{Agradecimientos}

Parte de esta investigación ha sido subvencionada por la ayuda para la contratación de personal investigador en formación predoctoral, Programa VALi+d (2014), concedida a Maria Vicent, así como el proyecto "Evaluación de la ansiedad escolar y su relación con variables psicoeducativas en la infancia. Estudio de la eficacia de un programa preventivo" (EDU2012-35124).

\section{REFERENCIAS}

Álvarez, A., Suárez, N., Tuero, E., Núñez, J.C., Valle, A., y Regueiro, B. (2015). Implicación familiar, autoconcepto del adolescente y rendimiento académico. European Journal of Investigation in Health Psychology and Education, 5(3), 293-311.

Barbaranelli, C., Caprara, G.V., Rabasca, A., y Pastorelli, C. (2003). A questionnaire for measuring the Big Five in late childhood. Personality and Individual Differences, 34, 645-664. 
Brannan, M.E., y Petrie, T.A. (2008). Moderators of the body dissatisfaction-eating disorder symptomatology relationship: replication and extension. Journal of Counseling Psychology, 55(2), 263-275. doi: 10.1037/0022-0167.55.2.263

Carrasco, M.A., Holgado, F.P., y Del Barrio, M.V. (2005). Dimensionalidad del cuestionario de los cinco grades (BFQ-N) en población infantil española. Psicothema, 17(2), 286-291.

Castro, J., Gila, A., Gual, P., Lahortiga, F., Saura, B., y Toro, J. (2004). Perfectionism dimensions in children and adolescents with Anorexia Nervosa. Journal of Adolescent Health, 35(5), 392-398. doi:10.1016/j.jadohealth.2003.11.094

Castro, M., Expósito-Casas, E., López-Martín, E., Lizasoain, L., Navarro-Asencio, E., y Gaviria, J.L. (2015). Parental involvement on student academic achievement: a meta-analysis. Educational Research Review, 14, 33-46. doi: 10.1016/j.edurev.2015.01.002

Cohen, J. (1988). Statistical power analysis for the behavioral sciences. Hillsdale, NJ: Erlbaum.

Cox, S.L., y Chen, J. (2014). Perfectionism: a contributor to social anxiety and its cognitive processes. Australian Journal of Psychology, 67(4), 231-240. doi: 10.1111/ajpy.12079

Cuttler, C., y Graf, P. (2007). Personality predicts prospective memory task performance: an adult lifespan study. Scandinavian Journal of Psychopathology, 48(3), 215-231. doi: 10.1111/j.1467-9450.2007.00570.x

De Cuyper, K., Claes, L., Hermans, D., Pieters, G., y Smits, D. (2015). Psychometric properties of the multidimensional perfectionism scale of hewitt in a dutch-speaking sample: associations with the Big Five personality traits. Journal of Personality Assessment, 97(2), 182-190. doi: 10.1080/00223891.2014.963591

De Maris, A. (2003). Logistic regression. En J.A. Schinka y W.F. Velicer (Eds.), Research methods in psychology (pp. 509-532). Nueva Jersey: John Wiley \& Sons.

Dekryger, N.A. (2005). Childhood perfectionism: measurement, phenomenology, and development (Tesis doctoral). Recuperada de la base de datos de ProQuest.

Dunkley, D.M., y Kyparissis, A. (2008). What is DAS self-critical perfectionism really measuring? Relations with the five-factor model of personality and depressive symptoms. Personality and Individual Differences, 44(6), 1295-1305. doi:10.1016/j.paid.2007.11.022

Enns, M.W., Cox, B.J., y Clara, I.P. (2005). Perfectionism and neuroticism: a longitudinal study of specific vulnerability and diathesis-stress models. Cognitive Therapy and Research, 29(4), 463-478. doi: 10.1007/s10608-005-2843-04

Flett, G.L., Hewitt, P.L., Boucher, D.J., Davidson, L.A., y Munro, Y. (2000). The ChildAdolescent Perfectionism Scale: development, validation, and association with adjustment. Manuscrito inédito. York University, Toronto, Ontario, Canadá.

Flett, G.L., Hewitt, P.L., Oliver, J.M., y Macdonald, S. (2002). Perfectionism in children and their parents: a developmental analysis. En G.L. Flett, y P.L. Hewitt (Eds.), Perfectionism: Theory, research, and treatment (pp. 89-132). Washington, DC: American Psychological Association.

Hill, R.W., McIntire, K., y Bacharach, V.R. (1997). Perfectionism and the Big Five Factors. Journal of Social Behavior and Personality, 12(1), 257-270.

Inglés, C.J., Gonzálvez, C., García-Fernández, J.M., Vicent, M., y Martínez-Monteagudo, M.C. (2015). Current status of research on school refusal. European Journal of Education and Psychology, 8(1), 37-52.

Miquelon, P., Vallerand, R.J., Groucet, F.M.E., y Cardinal, G. (2005). Perfectionism, academic motivation, and psychological adjustment: an integrative model. Personality and Social Psychology Bulletin, 31(7), 913-924. doi: 10.1177/0146167204272298

Molnar, D.S., Flett, G.L., Sadava, S.W., y Colautti, J. (2012). Perfectionism and health functioning in women with fibromyalgia. Journal of Psychosomatic Research, 73(4), 295-300. 
Molnar, D.S., Sadava, S.W., Flett, G.L., y Colautti, J. (2012). Perfectionism and health: a mediational analysis of the roles of stress, social support and health-related behaviours. Psychology and Health, 27(7), 846-864. doi: 10.1080/08870446.2011.630466

Morris, L., y Lomax, C. (2014) Review: assessment, development, and treatment of childhood perfectionism: a systematic review. Child and Adolescent Mental Health, 19(4), 225234. doi:10.1111/camh.12067

Nathanson, C., Paulhus, D.L., y Williams, K.M. (2006). Predictors of a behavioral measure of scholastic cheating: personality and competence but not demographics. Contemporary Educational Psychology, 31(1), 97-122. doi:10.1016/j.cedpsych.2005.03.001

O'Connor, D.B., y O'Connor, R.C. (2004). Perceived changes in food intake in response to stress: the role of conscientiousness. Stress and Health, 20(5), 279-291. doi: 10.1002/smi.1028

Perera, M.J. y Chang, E.C. (2015). Ethnic variations between Asian and European Americans in interpersonal sources of socially prescribed perfectionism: it's not just about parents! Asian American Journal of Psychology, 6(1), 31-37. doi: 10.1037/a0036175

Regueiro, B., Rodríguez S., Piñeiro, I., Estévez, I., Ferradás, M., y Suárez, N. (2015). Diferencias en la perfección de la implicación parental en los deberes escolares en función del nivel de motivación de los estudiantes. European Journal of Investigation in Health, Psychology and Education, 5(3), 313-323. doi: 10.1989/ejihpe.v5i3.134

Sherry, D.L., Sherry, S.B., Hewitt, P.L., Mushquash, A., y Flett, G.L. (2015). The existential model of perfectionism and depressive symptoms: tests of incremental validity, gender differences and moderated mediation. Personality and Individual Differences, 76, 104110. doi:10.1016/j.paid.2014.12.002

Sherry, S.B., y Hall, P.A. (2009). The perfectionism model of being eating: tests of an integrative model. Journal of Personality and Social Psychology, 96(3), 690-709.

Sherry, S.B., Hewitt, P.L., Sherry, D.L., Flett, G.L., y Graham, A.R. (2010). Perfectionism dimensions and research productivity in psychology professors implications for understanding the (mal)adaptiveness of perfectionism. Canadian Journal of Behavioural Science, 42(4), 273-283. doi: 10.1037/a0020466

Sherry, S.B., Hewitt, P.L., Flett, G.L., Lee-Baggley, D.L., y Hall, P.A. (2007). Trait perfectionism and perfectionistic self-presentation in personality pathology. Personality and Individual Differences, 42(3), 477-490. doi:10.1016/j.paid.2006.07.026

Stairs, A.M., Smith, G.T., Zapolski, T.C.B., Combs, J.L., y Settles, R. E. (2012). Clarifying the construct of perfectionism. Assessment, 19(2), 146-166.

Stoeber, J. (2014). Multidimensional perfectionism and the DSM-5 personality traits. Personality and Individual Differences, 64, 115-120. doi: 10.1016/j.paid.2014.02.031

Stoeber, J., Otto, K., y Dalbert, C. (2009). Perfectionism and the Big Five: conscientiousness predicts longitudinal increases in self-oriented perfectionism. Personality and Individual Differences, 47(4), 363-368. doi:10.1016/j.paid.2009.04.004

Valle, A., Regueiro, B., Rodríguez, S., Piñeiro, I., Freire, C., Ferradás, M., y Suárez, N. (2015). Perfiles motivacionales como combinación de expectativas de autoeficacia y metas académicas en estudiantes universitarios. European Journal of Education and Psychology, 8(1), 1-8.

Recibido: 17 de diciembre de 2015 Recepción Modificaciones: 21 de febrero de 2016 Aceptado: 23 de febrero de 2016 